\title{
ATUALIZAÇÃO E VALIDAÇÃO DE EQUIPAMENTO PARA ENSAIOS DE AMOLECIMENTO E FUSÃO DE CARGAS FERRÍFERAS*
}

\author{
Lucas Batista de Almeida ${ }^{1}$ \\ Ismael Vemdrame Flores ${ }^{2}$ \\ Maurício Covcevich Bagatini ${ }^{3}$
}

\begin{abstract}
Resumo
Durante o processo de redução das cargas ferrosas em altos-fornos, observa-se a formação de uma região responsável por cerca de $70 \%$ da perda de carga do altoforno, denominada zona coesiva ou zona de amolecimento e fusão, estruturada com camadas alternadas de coque metalúrgico e óxidos ferrosos pastosos e impermeáveis. Foram desenvolvidos ensaios para determinar as propriedades a altas temperaturas das cargas ferrosas, pela sua majoritária influência nas características da zona coesiva. Esse trabalho apresenta os parâmetros de ensaio e os equipamentos envolvidos e a metodologia de atualização de um equipamento para a avaliação das características de amolecimento e fusão das cargas ferrosas. Foram analisados ensaios de validação em diferentes amostras objetivando verificar a padronização, reprodutividade e repetibilidade do ensaio de amolecimento e fusão. Palavras-chave: Zona coesiva; Amolecimento e fusão de cargas ferrosas; Altoforno; Produção de ferro.
\end{abstract}

\section{UPDATING AND VALIDATION OF AN EQUIPMENT FOR SOFTENING AND MELTING OF IRON BERAING MATERIALS}

\begin{abstract}
During the reducing process of the iron bearing materials in blast furnaces, it is observed the formation of a region responsible for about $70 \%$ of the blast furnace pressure loss, called cohesive zone or softening and melting zone, which is structured in alternating layers of coke and impermeable softened iron oxides. Tests were developed to determine the high temperature properties of the iron bearing materials, since they have major influence on the characteristics of the cohesive zone. This work presents the test parameters and the equipment involved and the updating methodology of an equipment for the evaluation of the softening and melting characteristics of the iron bearing materials. Validation tests on different samples were carried out to verify the standardization, reproducibility and repeatability of the softening and melting tests.

Keywords: Cohesive Zone, Softening and Melting of iron bearing materials, blast furnace, Ironmaking.

1 Engenharia Metalúrgica, Graduando, UFMG, Departamento de Engenharia Metalúrgica e de Materiais, Belo Horizonte, Minas Gerais, Brasil.

2 Engenharia Metalúrgica, Doutorando, UFMG, Departamento de Engenharia Metalúrgica e de Materiais, Belo Horizonte, Minas Gerais, Brasil

3 Engenharia Metalúrgica, Doutor, Professor Adjunto e Coordenador do Laboratório de Processos Metalúrgicos, UFMG, Belo Horizonte, Minas Gerais, Brasil.
\end{abstract}




\section{Introdução}

O alto-forno é a principal tecnologia de redução empregada para a produção de ferro-gusa, sendo atualmente responsável por cerca de 95\% do ferro-gusa no mundo (World Steel Association, 2013). O processo de fabricação de ferro primário em altos-fornos é bastante complexo e começou a ser melhor compreendido e a partir dos trabalhos de resfriamento e dissecação realizados principalmente pelos japoneses (Kaushik [1], Sasaki [2] e Kanbara [3]). Nesses estudos, observou-se a formação de regiões distintas de acordo com as reações químicas, temperatura e estado de oxidação dos óxidos de ferro. Dentre essas, verificou-se a formação de uma região estruturada por camadas alternadas de carga metálica pastosa e impermeável e coque metalúrgico, conhecida como zona coesiva ou de amolecimento e fusão (Figura 1 - A).

De acordo com Higuchi [4], essa região é responsável por grande parte da perda de carga no interior do alto-forno, ou seja, é a região de menor permeabilidade à ascensão dos gases e por isso influencia fortemente na cinética de redução das cargas ferríferas, fato indicado na Figura 1 - B. Segundo Kaushik [1], as propriedades a alta temperatura das matérias-primas ferrosas (minério bitolado, sínter e/ou pelotas) majoritariamente controlam o formato, espessura e posição da zona coesiva. Sendo que essa última também pode ser afetada pela distribuição de carga, particularmente pela relação minério/coque, e pelo perfil de temperaturas do alto-forno.

(A

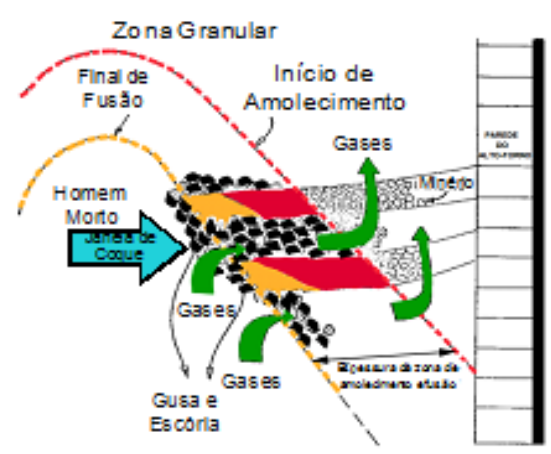

(B

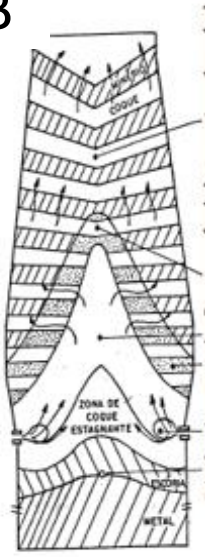

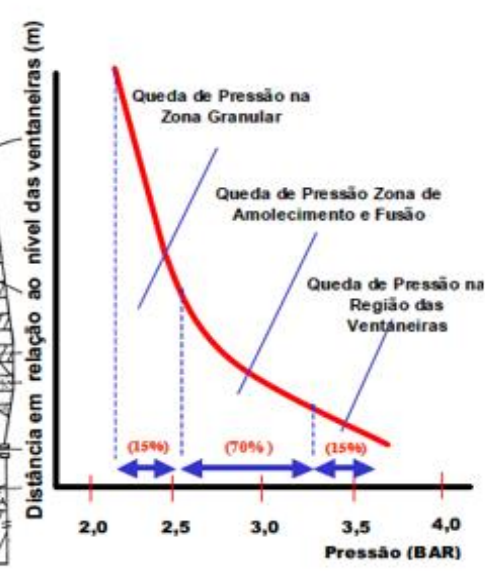

Figura 1: (a) Zona coesiva ou amolecimento e fusão; (b) Perda de pressão nas zonas de um alto-forno.

Devido à importância das propriedades a alta temperatura das matériasprimas ferrosas sobre as características da zona coesiva, diversos pesquisadores procuraram desenvolver ensaios que possam avaliar o comportamento desses materiais nas regiões inferiores dos altos-fornos. Porém, a diversidade de ensaios existentes na literatura, tanto em relação aos equipamentos utilizados, quanto às condições experimentais e variadas interpretações dos resultados obtidos tornam a padronização difícil e incentivam o desenvolvimento de tecnologias próprias. De acordo com Barnaba [6], a partir dos resultados de amolecimento e fusão e pelo entendimento de como as matérias-primas ferrosas podem afetar a zona coesiva, é possível obter um ganho de produtividade considerável para os altos-fornos. A formação de uma zona coesiva estreita e localizada em uma região adequada de um alto-forno gera economia na utilização de termorredutores e um aumento de 
produtividade devido à rápida fusão do ferro e da escória (menor perda de pressão associada à região de amolecimento e fusão).

Tendo em vista que nas últimas décadas a qualidade dos minérios de ferro e seus produtos vem decaindo consideravelmente, principalmente pelo aumento dos teores de ganga $\left(\mathrm{SiO}_{2}\right.$ e $\left.\mathrm{Al}_{2} \mathrm{O}_{3}\right)$ e pelo afinamento dos minérios, faz-se cada vez mais necessária a análise e previsão do comportamento de amolecimento e fusão das cargas ferríferas utilizadas em altos-fornos. Nesse contexto, o Laboratório de Processos Metalúrgicos da UFMG, o qual possui um equipamento voltado para esse tipo de análise, no entanto, inoperante, obteve a oportunidade de realizar a atualização do mesmo, visando à realização de estudos científicos na área, bem como auxiliar as empresas do setor siderúrgico no reconhecimento das propriedades das suas matérias-primas. Assim, esse trabalho tem como objetivo apresentar o equipamento de amolecimento e fusão, as mudanças realizadas para sua operação e os resultados preliminares obtidos a partir de testes com pelotas e sínter.

\section{Materiais e Métodos}

Para se realizar a reforma do equipamento, o qual possui diversos dispositivos e variáveis operacionais e resultantes dos ensaios, visando obter um ensaio reprodutivo, fez-se necessária uma revisão bibliográfica com intuito de entender o que ocorre durante o experimento, quais os parâmetros analisados e os resultados obtidos.

\subsection{Concepções Básicas}

Os equipamentos utilizados em ensaios de amolecimento e fusão usualmente consistem de um forno com aquecimento elétrico que pode atingir temperaturas de até $1600^{\circ} \mathrm{C}$, um aplicador de carga sobre a amostra, um sistema de controle de vazão e composição de gás que permeia no leito, sensores para mensurar a contração e perda de pressão do leito de amostra e um sistema de aquisição de dados.

Durante experimentos de amolecimento e fusão, busca-se reproduzir em laboratório as condições que mais se aproximam do que realmente acontece em um alto-forno. A partir de trabalhos realizados por Rocha [7], Ritz [8], Castro [5] e Iljana [9] nota-se que as metodologias para a avaliação das propriedades em altas temperaturas de cargas metálicas possuem os seguintes pontos em comum:

- Redução da carga ferrosa previamente ao estágio de amolecimento da carga

- Perfil de temperatura

- Atmosfera gasosa

- Velocidade do gás no leito

- Solicitações mecânicas a que a carga ferrosa deve ser submetida.

De acordo com Castro [5], a manipulação adequada do perfil de temperatura e composição gasosa permite simular a redução indireta (gás/sólido) de forma similar ao que acontece na região superior dos altos-fornos. Outro ponto em comum nesse tipo ensaio é a presença de material redutor (usualmente coque) juntamente com a carga ferrosa. Graças a isso é possível também simular o mecanismo de redução direta (sólido/líquido) a que o material portador de ferro é submetido a partir da zona de amolecimento e fusão.

Durante o experimento, a avaliação das características da zona coesiva é realizada através da análise dos seguintes parâmetros: contração do leito e perda de 
pressão (diferença entre entrada e saída) do gás que permeia a amostra. Ambos os parâmetros são acompanhados em função da temperatura do forno, permitindo acompanhar a evolução do fenômeno de amolecimento e fusão e a determinação de suas temperaturas características. Alguns equipamentos ainda realizam a análise química continua do gás de saída, quantificando os teores de $\mathrm{CO}$ e $\mathrm{CO}_{2}$. Essa análise contribui principalmente na obtenção do grau de redução da amostra em função da temperatura de teste.

\subsection{Parâmetros de Ensaio e Reformas Envolvidas}

Devido à alta complexidade dos equipamentos de amolecimento e fusão (alta temperatura, partes móveis, entre outras), nota-se que não há uma padronização ou norma internacional para a realização desses ensaios. A Tabela 1 apresenta uma revisão dos principais parâmetros utilizados por diversos centros de pesquisa em experimentos de amolecimento e fusão.

Tabela 1: Parâmetros de ensaio de diversos centros de pesquisa e empresas.

\begin{tabular}{|c|c|c|c|c|c|c|c|c|c|}
\hline \multirow[b]{2}{*}{$\begin{array}{c}\text { Laboratoriol } \\
\text { Origem }\end{array}$} & \multicolumn{3}{|c|}{ Amoatra $\theta$ Cadinho } & \multicolumn{3}{|c|}{ Aquecimento } & \multicolumn{2}{|c|}{ Atmoafera Gagosa } & \multirow{2}{*}{$\begin{array}{l}\text { Carga } \\
\text { Kgricm }\end{array}$} \\
\hline & $\begin{array}{c}\begin{array}{c}\text { Masag/ } \\
\text { Altura } \\
\text { de } \\
\text { Amogtra }\end{array} \\
\end{array}$ & $\begin{array}{c}\text { Granulometrla } \\
\text { (mm) }\end{array}$ & $\begin{array}{l}\text { Dlámetro } \\
\text { do } \\
\text { cadilnho } \\
\text { (mm) }\end{array}$ & $\underset{\max _{(\mathrm{C})}^{\mathrm{T}}}{\mathrm{T}}$ & $\begin{array}{l}\text { Falxa } \\
\text { Temp. } \\
\left({ }^{2} \mathrm{C}\right)\end{array}$ & $\begin{array}{l}\text { Taxa } \\
\left({ }^{\circ} \mathrm{C}\right)\end{array}$ & Composiçao & $\begin{array}{c}\text { VazBol } \\
\text { Velocidade } \\
\text { do Gas }\end{array}$ & \\
\hline $\begin{array}{c}\text { BHP- } \\
\text { Australia } \\
\text { (Controle de } \\
\text { Qualidade) }\end{array}$ & $87 \mathrm{~g}$ & $\begin{array}{l}-12,5 \\
+9,7\end{array}$ & 42 & 1600 & Toda & 10 & $\begin{array}{c}30 \% \mathrm{CO} \text { e } \\
70 \% \mathrm{~N}_{2}\end{array}$ & $7,2 \mathrm{dU}$ min & 1,0 \\
\hline NSC - Japã̃o & $\begin{array}{l}\text { Massea } \\
\text { variável } \\
\text { f(carga) }\end{array}$ & $\begin{array}{l}-15,0 \\
+10,0\end{array}$ & 100 & 1650 & $\begin{array}{c}200- \\
600 \\
600- \\
900 \\
900- \\
1200 \\
>1200\end{array}$ & $\begin{array}{c}10 \\
4,3 \\
2 \\
5\end{array}$ & $\begin{array}{l}30 \% \mathrm{CO} \text { e } \\
70 \% \mathrm{NL}^{2}\end{array}$ & $\begin{array}{l}8 \mathrm{~cm} / \mathrm{s} \\
\text { (CNTP) }\end{array}$ & 1,0 \\
\hline $\begin{array}{l}\text { SGA-- } \\
\text { Nernanha }\end{array}$ & $\begin{array}{l}\text { Massa } \\
\text { variável } \\
\text { f(carga) }\end{array}$ & $\begin{array}{l}-12,5 \\
+10,0\end{array}$ & 80 & 1550 & $\begin{array}{c}20-450 \\
450- \\
900 \\
900 \\
900- \\
1560\end{array}$ & $\begin{array}{c}5 \\
10 \\
\{100 \text { min }) \\
5\end{array}$ & $\begin{array}{c}\text { Alternahncia } \\
\text { nas } \\
\text { proporōoses de } \\
\mathrm{CO}, \mathrm{CO} \text { e N2 }\end{array}$ & $30 \mathrm{~N} / \mathrm{min}$ & 1,0 \\
\hline $\begin{array}{l}\text { Kawasaki } \\
\text { Steel - } \\
\text { Japāo } \\
\text { (Pesquisa) }\end{array}$ & $\begin{array}{l}1000 \mathrm{~g} \\
\text { (sinter) }\end{array}$ & $\begin{array}{c}-15,0 \text { a }+10,0 \\
\text { (sinter) } \\
-13,0 \text { a } 10,0 \\
\text { (pelota) }\end{array}$ & 100 & 1500 & $\begin{array}{l}200- \\
800 \\
800- \\
1000 \\
1000- \\
1560\end{array}$ & $\begin{array}{l}5 \\
2 \\
5\end{array}$ & $\begin{array}{c}\text { Alternahncia } \\
\text { nas } \\
\text { proparçōes de } \\
\mathrm{CO}, \mathrm{CO} \text { e N2 }\end{array}$ & $30 \mathrm{NUmin}$ & $\begin{array}{c}0-1,0 \\
\left(<960^{\circ} \mathrm{C}\right) \\
1,0 \\
\left(>960^{\circ} \mathrm{C}\right)\end{array}$ \\
\hline $\begin{array}{c}\text { British Steel } \\
\text { - England } \\
\text { (HSTM) }\end{array}$ & $\begin{array}{c}\text { Mases } \\
\text { variável } \\
\text { f(carga)) }\end{array}$ & $\begin{array}{l}-12,5 \\
+10,0\end{array}$ & 60 & 1550 & $\begin{array}{l}2700 \\
700- \\
1560 \\
\end{array}$ & $\begin{array}{l}3,3 \\
6,6\end{array}$ & $\begin{array}{c}\text { Alternància } \\
\text { nas } \\
\text { proporcōes de } \\
\mathrm{CO} \mathrm{CO} \text { e Nz }\end{array}$ & $15 \mathrm{dV} / \mathrm{min}$ & 1,0 \\
\hline $\begin{array}{l}\text { Dofasco - } \\
\text { Canada }\end{array}$ & $500 \mathrm{~g}$ & $\begin{array}{r}-12,7 \\
+9,5\end{array}$ & 75 & 1800 & Toda & 5 & $\begin{array}{c}\text { Atternancia } \\
\text { nas } \\
\text { proporçōes de } \\
\text { CO. } \mathrm{CO}_{2} \text { e Nz }\end{array}$ & $20 \mathrm{dU} / \mathrm{min}$ & $\begin{array}{c}0,5 \text { ou } \\
1,0\end{array}$ \\
\hline Kaushik [1] & 6 pelotas & - & $\begin{array}{c}2 \text { pelotas } \\
\text { por } \\
\text { camada }\end{array}$ & - & Toda & 3 & $\mathrm{~N}_{2}$ & - & 1,0 \\
\hline Cloutby [10] & $\begin{array}{l}\text { Massea } \\
\text { variável } \\
\text { f(carga) }\end{array}$ & - & 60 & - & $\begin{array}{l}<700 \\
>700\end{array}$ & $\begin{array}{l}3,33 \\
6,67\end{array}$ & $\begin{array}{c}\text { Alternahneia } \\
\text { nas } \\
\text { proporcōes de } \\
\mathrm{CO}, \mathrm{CO} \text { e e N2 }\end{array}$ & $=$ & 0,5 \\
\hline $\begin{array}{c}\text { Nishimural } \\
\text { [11] }\end{array}$ & $\begin{array}{l}\text { Algumas } \\
\text { cerntenas } \\
\text { de } \\
\text { gramas }\end{array}$ & - & 85 & 1550 & $\begin{array}{l}<1000 \\
>1000\end{array}$ & $\begin{array}{c}5 \\
10\end{array}$ & $\begin{array}{c}29,4 \% \mathrm{CO} \\
5 \% \mathrm{Hze} \\
67,1 \% \mathrm{~N} 2\end{array}$ & - & $\begin{array}{c}1,0 \\
\left(\times 100^{\circ} \mathrm{C}\right)\end{array}$ \\
\hline
\end{tabular}

Observa-se que, apesar da não normatização do ensaio de amolecimento e fusão, diversos dos parâmetros utilizados por outros pesquisadores são semelhantes, como composição gasosa, temperatura máxima e granulometria. Sabendo-se dessas tendências e das capacidades do equipamento presente no laboratório para realização do ensaio, a seguir são definidos os parâmetros operacionais e as reformas necessárias para que o mesmo voltasse a operar adequadamente. 


\subsubsection{Composição e Vazão do Gás de Ensaio}

Para inertização do sistema, entre a temperatura ambiente e $800^{\circ} \mathrm{C}$, utilizouse uma vazão de nitrogênio de $4 \mathrm{~L} / \mathrm{min}$. Ao atingir-se $800^{\circ} \mathrm{C}$, a composição do gás foi alterada para uma mistura constituída por $30 \%$ monóxido de carbono e $70 \%$ de nitrogênio e vazão total de $8 \mathrm{~L} / \mathrm{min}$. A vazão utilizada propicia velocidades de gás de $7,4 \mathrm{~cm} / \mathrm{s}$, semelhante as observadas na literatura para outros equipamentos. Um sistema de gases com controle elétrico e um homogeneizador de mistura gasosa são utilizados para o fornecimento de gás para o ensaio.

\subsubsection{Pressão Mecânica Aplicada a Amostras}

A pressão mais comumente utilizada nesse tipo de ensaio e que foi adotada é a de $1 \mathrm{kgf} / \mathrm{cm}^{2}$, carga sendo aplicada a partir do instante em que o forno atinge $800^{\circ} \mathrm{C}$. Essa escolha é devido à intenção de reproduzir as condições encontradas em altos-fornos. Para realizar a aplicação de carga, foi realizada a atualização do sistema pneumático preexistente, a aquisição de um pistão pneumático cilíndrico com pressão de ar de trabalho máxima de 10 bar e a instalação de um controlador de pressão tipo PID, que permite o acionamento e variação da pressão do pistão durante qualquer instante do ensaio.

\subsubsection{Perfil Térmico do Ensaio}

A temperatura máxima de ensaio foi definida em $1580^{\circ} \mathrm{C}$, com o intuito de elevar ao máximo a temperatura no centro da amostra durante o ensaio, valor aproximado de $1500^{\circ} \mathrm{C}$. Como a redução da carga ferrosa é realizada durante o próprio ensaio, foi escolhido um perfil de aquecimento no qual a redução da carga anterior ao inicio de amolecimento atingi-se valores entre 60 e $80 \%$. O perfil de aquecimento adotado possui as seguintes etapas: aquecimento a $10^{\circ} \mathrm{C} / \mathrm{min}$ até atingir $1000^{\circ} \mathrm{C}$; aquecimento a $5^{\circ} \mathrm{C} / \mathrm{min}$ até atingir $1580^{\circ} \mathrm{C}$; patamar a $1580^{\circ} \mathrm{C}$ por 10 minutos.

Para se realizar o ensaio com o perfil térmico detalhado foi necessária a parcial reforma do forno resistivo já existente. Foi realizada a troca de três elementos resistivos de dissiliceto de molibdênio, adequação do circuito elétrico ligado ao forno, instalação de uma unidade de potência nova com corrente de trabalho de até $60 \mathrm{~A} \mathrm{e}$ compra de controlador do tipo PID que permite a programação e execução dos programas de aquecimento desejados.

Obteve-se, após revisão dos dados da literatura, uma sequência cronológica de ensaio representada graficamente através da Figura 2, onde é demonstrado o perfil térmico adotado. As marcações no perfil indicam etapas importantes do experimento, onde as variáveis de ensaio são alteradas. 


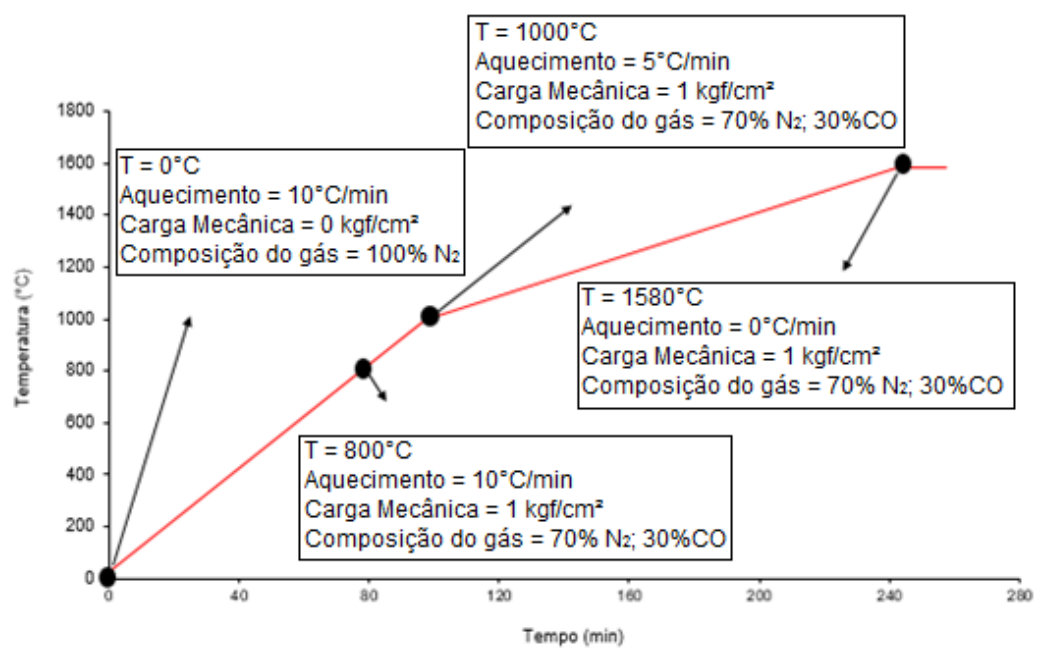

Figura 2: Perfil térmico do ensaio de redução a altas temperaturas sob carga.

\subsubsection{Preparação da Amostra}

As amostras utilizadas nos ensaios de amolecimento e fusão, sejam coque ou minério de ferro e seus produtos, são utilizadas na granulometria entre 10 e 12,5 $\mathrm{mm}$. O carregamento do cadinho (diâmetro $x$ altura) é realizado em camadas alternadas: duas camadas de coque com espessura de $20 \mathrm{~mm}$ e uma camada central de material ferroso com espessura de $40 \mathrm{~mm}$ (Figura 3). Essa especificação é considerada mais próxima das condições de carregamento de um alto-forno.

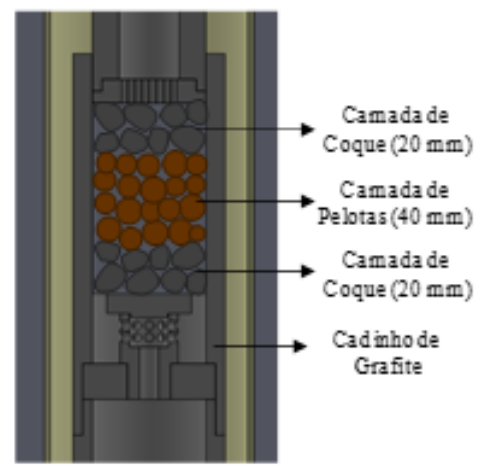

Figura 3: Cadinho de grafite esquemático com montagem de amostra.

\subsubsection{Sistema de Monitoramento e Aquisição de Dados}

Afim de se obter os parâmetros que permitem avaliar as propriedades de amolecimento e fusão das cargas ferrosas é necessária a coleta de dados relacionados ao deslocamento, pressão diferencial do leito e temperatura. O sensor de deslocamento existente encontrava-se em bom estado, sendo necessária apenas a aquisição de um sensor de pressão diferencial capacidade até 1 bar e precisão de 0,001 bar. Além disso, uma central de aquisição de dados (FieldLogger) foi instalada, permitindo a aquisição e o monitoramento dos dados e parâmetros do ensaio em tempo real. A Figura 4 mostra o forno de amolecimento e fusão atualizado e montado, sendo seus principais componentes indicados. 


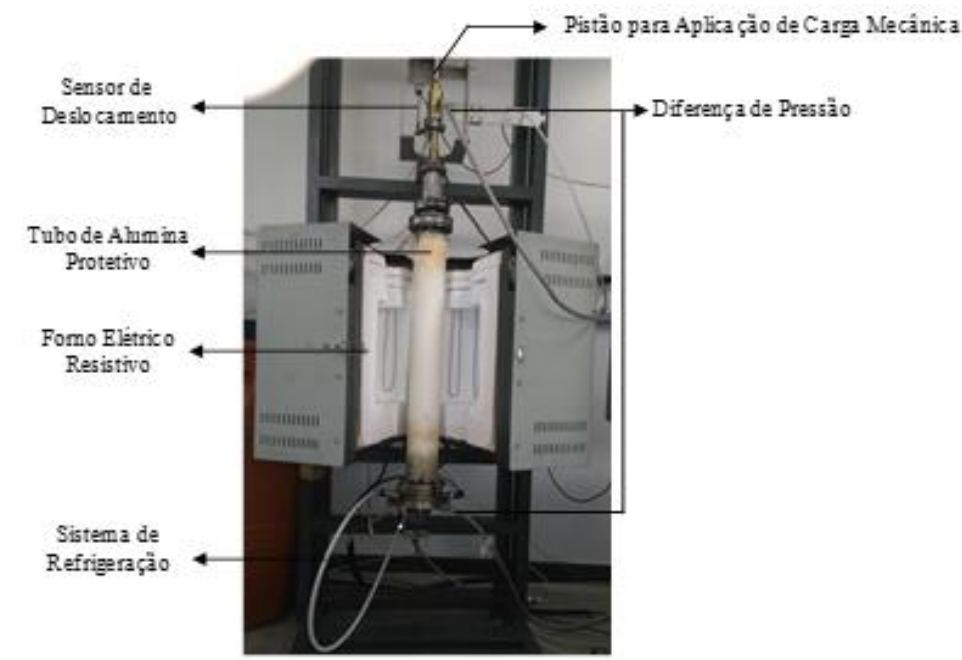

Figura 4: Visão frontal do equipamento de amolecimento e fusão seus principais componentes.

\subsection{Fenômeno de Amolecimento e Fusão}

Afim de compreender o fenômeno de amolecimento e fusão, Bakker [12] realizou ensaios laboratoriais que revelaram uma sequência de eventos típica. A Figura 5 ilustra os quatro estágios que a carga ferrífera passa durante o ensaio a altas temperaturas.

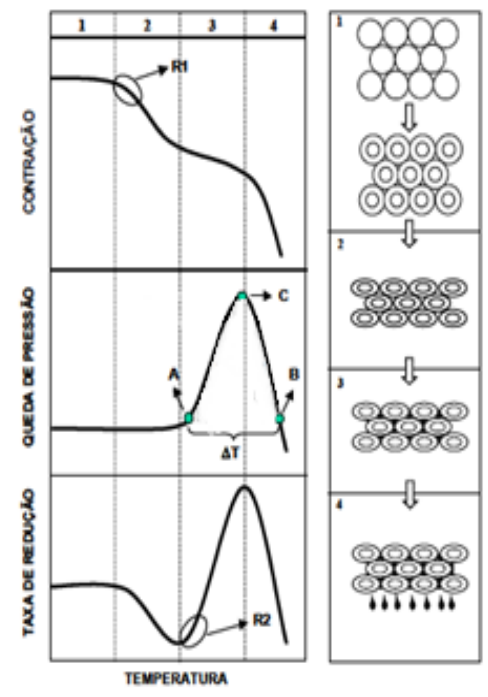

Figura 5: Estágios de amolecimento e fusão em relação as curvas de contração, perda de pressão e taxa de redução. (Castro [5]).

Durante o estágio 1 , anterior ao amolecimento, a carga ferrosa é inicialmente pré-reduzida ao nível da wustita. Após o período que corresponderia ao tempo de permanência nas zonas de reserva térmica e química, condições termodinâmicas favorecem a formação de uma camada externa de ferro metálico. Assim, forma-se, ao entorno do núcleo oxidado, uma camada de ferro metálico que posteriormente passa por uma etapa de densificação, podendo ocasionar pequena contração do leito.

No segundo estágio de amolecimento, ocorre uma diminuição na taxa de redução acompanhada por uma contração significativa do leito. A perda de pressão não se eleva apesar da contração, uma vez que o leito ainda apresenta boa 
permeabilidade. A deformação que ocorre é devido a formação de fase líquida no interior das partículas.

Durante o terceiro estágio, ocorre a exsudação do líquido (escória primária rica em $\mathrm{FeO}$ ) presente no interior das partículas, que migra para a macroporosidade do leito. Com isso, a taxa de redução aumenta significativamente devido ao contato direto da escória com gases redutores e coque. A redução do $\mathrm{FeO}$ da escória faz com que sua temperatura de fusão aumente, causando a solidificação do material. Percebe-se então a elevação abrupta da queda de pressão devido à redução da permeabilidade do leito.

No estágio final de fusão, inicia-se o gotejamento de fase líquida, composta por ferro metálico, escória ou ambos, que resulta em uma grande contração do leito, sendo essa dependente da fração de líquidos formada nessa faixa de temperatura. A queda de pressão do leito e a diminuição gradual da taxa de redução estão associadas ao gotejamento da fase líquida.

\subsection{Parâmetros Derivados do Ensaio de Amolecimento e Fusão}

Autores como Nogueira [13], Bakker [12], Ritz [8] e Barnaba [6] discutem sobre os parâmetros considerados relevantes na avaliação de propriedades em altas temperaturas. Constata-se que a literatura apresenta coerência entre os índices considerados como importantes para avaliação destas propriedades, sendo que os de uso mais frequente pelas instituições que fazem este tipo de avaliação são:

- Temperatura de início de amolecimento: relacionada à temperatura na qual a contração do leito de carga ferrosa tem início. Associada a uma contração de $10 \%$ do leito. Usualmente a queda de pressão no leito não apresenta valores significativos. Essa temperatura situa-se na fronteira dos estágios 1 e 2, na região R1 (Figura 5).

- Temperatura de amolecimento: definida como a temperatura na qual a contração do leito atinge $50 \%$ da altura inicial da carga metálica. Ritz [8] relaciona esta temperatura característica do ensaio como um índice de controle da evolução da contração do leito e também a convencionam como uma referência para a temperatura de amolecimento.

- Temperatura de início da zona coesiva: identificada como a temperatura na qual a queda de pressão no leito começa a se elevar de maneira significativa ( $>200 \mathrm{~mm} \mathrm{H} \mathrm{H}_{2} \mathrm{O}$ ) passando a ser controlada pela crescente presença de líquido no mesmo. Este ponto situa-se no início do estágio 3 nas proximidades do ponto A na Figura 5.

- Temperatura de final da zona coesiva: usualmente definida como sendo a temperatura na qual a queda de pressão no leito retorna aos níveis apresentados no amolecimento e início da zona coesiva. Analisando a Figura 5, pode-se localizar esta temperatura na região final de fusão no estágio 4, próximo ao ponto $\mathrm{B}$.

- Intervalo de amolecimento: neste intervalo de temperatura a fração de vazios do leito da amostra diminui gradualmente com consequente retardamento da taxa de redução. No entanto, a queda de pressão experimentada pelo leito ainda permite a redução da carga seja feita predominantemente pelo gás redutor.

- Intervalo de temperatura da zona coesiva: tem como objetivo medir de maneira indireta e qualitativa da espessura da zona coesiva do alto-forno. Corresponde à diferença entre as temperaturas nas quais a queda de pressão saiu de níveis considerados baixos, passou pelo máximo valor de 
queda de pressão e retornou a valores baixos. O valor desta diferença está associado ao gradiente de temperatura experimentado pela carga metálica na zona coesiva do alto-forno. Usualmente de o intervalo de temperatura da zona coesiva corresponde à diferença entre a temperaturas correspondentes aos pontos A e B da Figura 5.

\subsection{Ensaios de Validação do Forno de Amolecimento e Fusão}

De forma a confirmar experimentalmente a funcionalidade e repetibilidade do equipamento atualizado, foram realizados ensaios de validação com a metodologia apresentada no item 2.2 e utilizando uma amostra de pelota e uma de sínter. As amostras de pelota e sínter foram ensaiadas em triplicata e duplicata, respectivamente. A composição química das amostras é apresentada na Tabela 2. Obteve-se como resultado direto do ensaio o deslocamento das cargas e a perda de pressão no leito com o andamento do ensaio e, a partir destes dados, obtiveram-se os parâmetros relevantes para avaliação das propriedades a altas temperaturas citadas no item 2.4 .

Tabela 2: Composição química das amostras ensaiadas para validação do equipamento.

\begin{tabular}{cccccccccc}
\hline Material & $\% \mathrm{Fe}$ & $\% \mathrm{FeO}$ & $\% \mathrm{SiO}_{2}$ & $\% \mathrm{CaO}$ & $\% \mathrm{Al}_{2} \mathrm{O}_{3}$ & $\% \mathrm{P}$ & $\% \mathrm{~S}$ & $\% \mathrm{MgO}$ & $\% \mathrm{TiO}_{2}$ \\
\hline Pelota & 65,80 & - & 2,45 & 2,65 & 0,60 & 0,030 & 0,003 & - & - \\
\hline Sínter & 56,76 & 8,05 & 6,02 & 9,79 & 1,42 & 0,035 & - & 1,45 & 0,083 \\
\hline
\end{tabular}

\section{Resultados e Discussão}

\subsection{Ensaios de Validação}

As Figuras 6 e 7 apresentam as curvas de contração e de perda de pressão do gás durante os ensaios com pelotas e sínters, respectivamente. A Tabela 3 apresenta os parâmetros derivados dessas curvas em termos de média e desvio padrão, obtidos como resultados dos ensaios de validação.

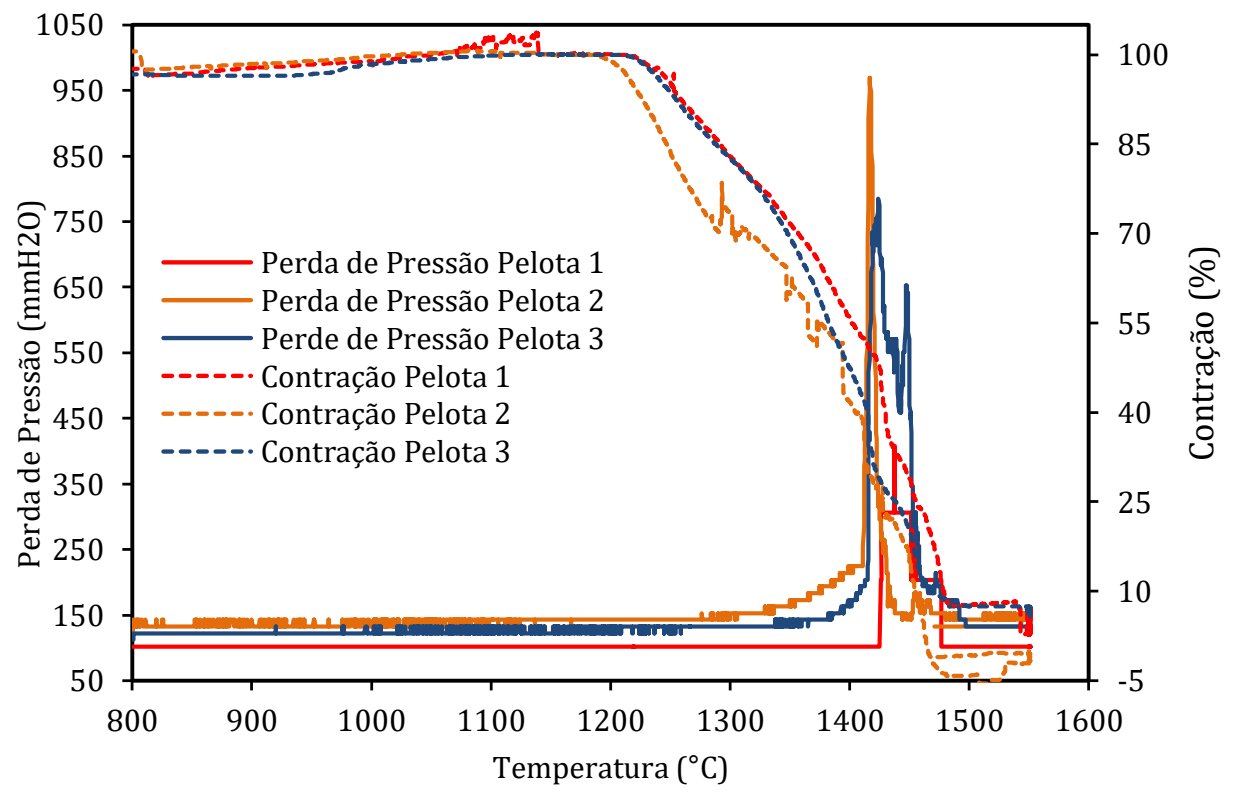

Figura 6: Curvas de perda de pressão e contração do leito durante os ensaios de amolecimento e fusão realizados com pelotas. 


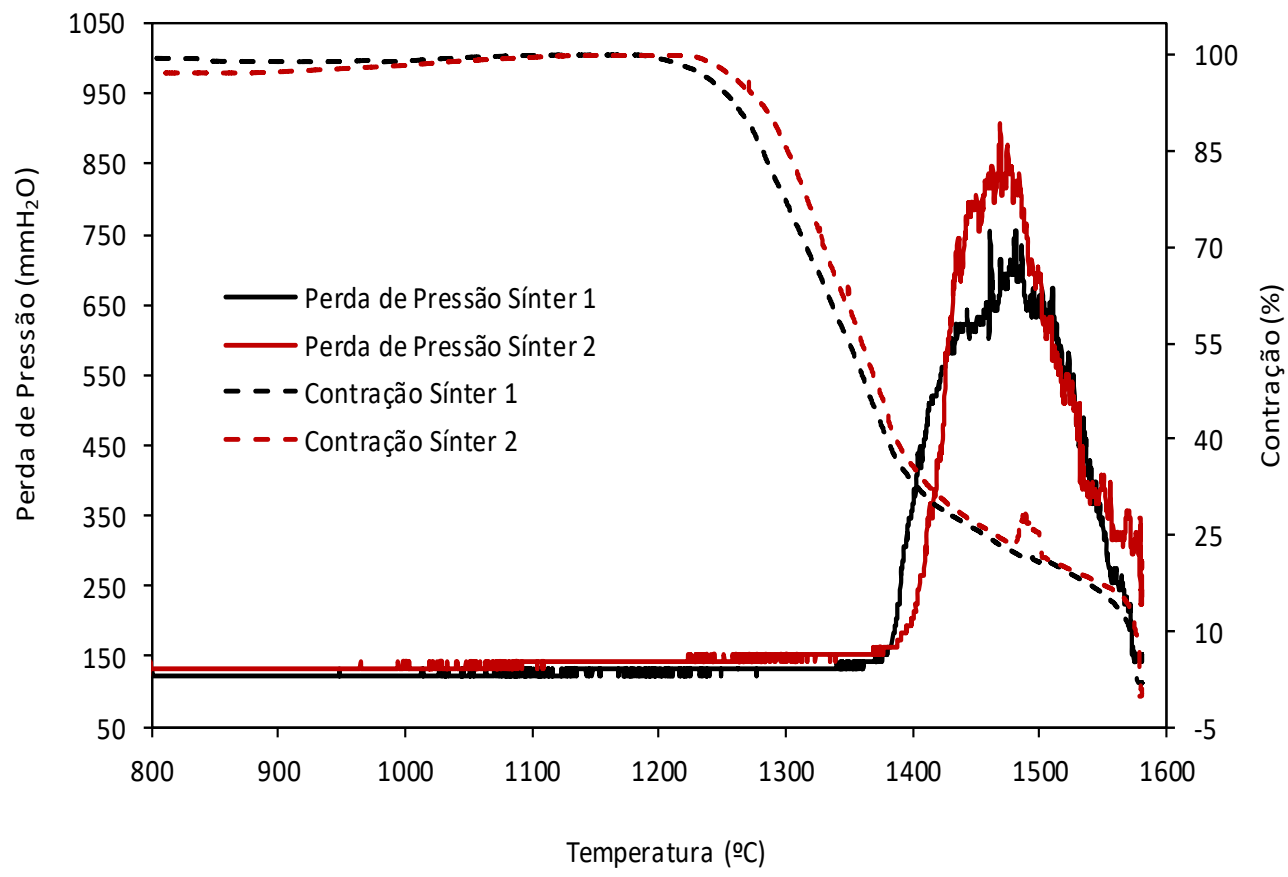

Figura 7: Curvas de perda de pressão e contração do leito durante os ensaios de amolecimento e fusão realizados com sínteres.

Tabela 4: Média e desvio padrão dos parâmetros de avaliação dos comportamentos de amolecimento e fusão das cargas ferrosas ensaiadas.

\begin{tabular}{lcccc}
\hline Parâmetros & \multicolumn{2}{c}{ Pelotas } & \multicolumn{2}{c}{ Sínteres } \\
& Média & $\begin{array}{c}\text { Desvio } \\
\text { Padrão }\end{array}$ & Média & $\begin{array}{c}\text { Desvio } \\
\text { Padrão }\end{array}$ \\
\hline Temperatura de Início de Amolecimento $\left({ }^{\circ} \mathrm{C}\right)$ & 1248 & 19 & 1279 & 14 \\
\hline Temperatura de Amolecimento $\left({ }^{\circ} \mathrm{C}\right)$ & 1402 & 14 & 1365 & 6 \\
\hline Temperatura de Início da Zona Coesiva $\left({ }^{\circ} \mathrm{C}\right)$ & 1408 & 20 & 1404 & 11 \\
\hline Temperatura Final da Zona Coesiva $\left({ }^{\circ} \mathrm{C}\right)$ & 1457 & 23 & 1554 & 1 \\
\hline Máxima Perda de Pressão $(\mathrm{mmH} \mathrm{H})$ & 721 & 286 & 831 & 108 \\
\hline Temperatura de Máxima Perda de Pressão $\left({ }^{\circ} \mathrm{C}\right)$ & 1426 & 10 & 1465 & 6 \\
\hline Intervalo de Amolecimento $\left({ }^{\circ} \mathrm{C}\right)$ & 209 & 12 & 86 & 8 \\
\hline Intervalo da Zona Coesiva $\left({ }^{\circ} \mathrm{C}\right)$ & 49 & 3 & 150 & 7 \\
\hline Intervalo de Amolecimento e Fusão $\left({ }^{\circ} \mathrm{C}\right)$ & 209 & 12 & 275 & 10 \\
\hline
\end{tabular}

As curvas obtidas (Figuras 6 e 7 ) apresentaram o comportamento usualmente observado para ensaios de amolecimento e fusão. No decorrer do ensaio e com o aumento da temperatura, nota-se inicialmente um aumento do volume do leito, devido à expansão térmica da carga ferrosa e coque. A partir de aproximadamente $1200^{\circ} \mathrm{C}$ inicia-se a contração das amostras, que ocorre continuamente até 0 final do ensaio. A pressão diferencial se mantém estável durante o ensaio até o instante em que aumenta rapidamente a partir de $1400^{\circ} \mathrm{C}$, até atingir um valor máximo em temperaturas em torno de 1426 e $1465^{\circ} \mathrm{C}$, respectivamente para pelotas e sínter, devido a presença de líquido no leito. Após esse ponto máximo, os valores de perda de pressão decrescem até atingirem valores próximos aos observados inicialmente, devido ao gotejamento das fases líquidas fundidas que permite a passagem do gás pela amostra. As informações com relação aos formatos das curvas obtidas como resposta e os fenômenos ocorridos 
durante um ensaio de amolecimento e fusão correspondem aos que foram citados no item 2.3 e que são encontrados na literatura.

Pôde-se perceber uma variação significativa no valor de perda de pressão máxima entre os ensaios, especialmente para as pelotas. Essa variação é justificada por problemas de junção de peças de grafite que fazem parte do conjunto do cadinho. A medida que os ensaios foram executados, correções foram realizadas, se obtendo desvios padrões significativamente melhores para as amostras de sínter.

A grande variação apresentada no intervalo de amolecimento e fusão quando se altera a carga metálica de sínter para pelota, deve estar relacionada as diferenças fundamentais de composição química, porosidade e redutibilidade entre esses materiais. Foi possível notar um maior intervalo de amolecimento nas pelotas (Tabela 4), esse fato pode ser explicado pela maior porosidade e maior resistência da casca metálica formada para as pelotas. Porém nota-se também que os sínters apresentaram uma zona coesiva maior, devido a sua maior basicidade e diferença de viscosidade das fases líquidas formadas na zona coesiva em relação às pelotas. Porém para ambas as cargas se percebem tendências nos resultados obtidos, comprovando mais uma vez a reprodutividade dos resultados obtidos.

Nos ensaios com pelotas e sínters, procura-se avaliar as características de amolecimento para a mesma matéria-prima em condições as mais próximas possíveis. Os resultados indicaram a repetibilidade do ensaio, apresentando desvios padrões para os parâmetros de avaliação das propriedades de amolecimento e fusão de no máximo $23^{\circ} \mathrm{C}$ e em média de $13.5^{\circ} \mathrm{C}$. Rocha [14] apresenta desvios padrões de aproximadamente $25^{\circ} \mathrm{C}$ para os intervalos de amolecimento e da zona coesiva, o que valida a repetibilidade dos ensaios realizados.

Para aumentar a qualidade de ensaios futuros, busca-se a diminuição dos desvios padrões obtidos para os parâmetros de resposta. Para isso deve-se atuar na vedação do conjunto do cadinho e da montagem externa e na aquisição de um sistema de análise do gás de saída, permitindo acompanhar o grau de redução das amostras durante o ensaio.

\subsection{Atualização do Equipamento}

Foi constatado a partir dos experimentos realizados e resultados obtidos que as atualizações e reformas realizadas no forno de amolecimento e fusão foram satisfatórias. O sistema de aquecimento do forno, com novas resistências, unidade de potência e controlador, possibilitou que se atingisse as temperaturas e rampas de aquecimento desejadas. O sistema pneumático utilizado, junto aos novos pistão e controlador adquiridos propiciaram um controle e nível de carga adequado para a realização dos ensaios. O sistema de gases utilizado também se mostrou dentro do esperado, garantindo a composição e homogeneidade do gás em cada etapa do experimento.

Quanto aos sensores de deslocamento e diferença de pressão utilizados, ambos apresentaram faixa de trabalho dentro do necessário para a execução dos ensaios. Entretanto, num primeiro ensaio, o sistema de aquisição de dados se mostrou inadequado, obtendo dados com baixa precisão (Figura 6, curva de perda de pressão pelota 1). Após esse ensaio, medidas de correção foram realizadas, de forma que nos experimentos subsequentes, o sistema de monitoramento e aquisição de dados foi capaz de coletar os parâmetros de interesse com a precisão e intervalos de tempo necessários. 


\section{Conclusão}

O presente trabalho apresenta a atualização realizada em um equipamento para determinação de propriedades de amolecimento e fusão de matérias-primas ferrosas, bem como realizar ensaios de validação para o mesmo.

A partir dos resultados obtidos, pode-se constatar que a atualização realizada no equipamento foi suficiente para sua adequação. As mudanças realizadas permitiram a obtenção de um perfil de aquecimento adequado, bem como a aplicação de uma carga constante sobre o leito de partículas sendo ensaio. $O$ sistema de aquisição e monitoramento de dados se mostrou capaz de acompanhar os parâmetros de temperatura, deslocamento e perda de pressão ao longo do ensaio.

As curvas obtidas a partir dos ensaios de validação apresentaram o comportamento esperado pela literatura. A amostra de pelotas, em comparação a um sínter industrial, iniciou o seu amolecimento em temperaturas mais baixas, tendo maior intervalo de amolecimento e menor intervalo de zona coesiva. Além disso, os resultados de validação apresentaram desvios padrões satisfatórios entre as repetições de cada amostra, tendo boa repetibilidade e representatividade.

\section{Agradecimentos}

Os autores agradecem ao Conselho Nacional de Desenvolvimento Científico e Tecnológico (CNPq) e a Coordenação de Aperfeiçoamento de Pessoal de Nível Superior (CAPES) pelo incentivo financeiro.

\section{Referências}

1) Kaushik $P$, Fruehan RJ. Mixed burden softening and melting phenomena in blast furnace operation Part $1-\mathrm{X}$-ray observation of ferrous burden. Ironmaking and Steelmaking. 2006, 33(6): 507-519.

2) Sasaki K, Nakatani F, Hatano M, Watanabe M, Shimoda T, Yokotani K, et al..Transactions Iron Steel Institute. 1977, 17: 391-400.

3) Kanbara K, Hagiwara T, Shigemi A, Kondo S, Kanayama Y, Wakabayashi K, et al. Transactions Iron Steel Institute. 1977, 17: 371-380.

4) Higuchi M. Improvement of flow in large blast furnaces. Proceedings of Blast. Furnace Aerodynamics Symposium. Wollongong, 1975: 129-135.

5) Castro AA. Avaliação de Propriedades em Altas Temperaturas de Pelotas para Alto-Forno (dissertação de mestrado). Ouro Preto: Universidade Federal de Ouro Preto, 2004.

6) Barnaba P. Influência das características químicas nas propriedades de amolecimento e fusão do sinter de minério de ferro. Metalurgia - ABM. 1986, 42(345).

7) Rocha GT. Estudo básico sobre o amolecimento dos constituintes de carga metálica do alto-forno (dissertação de mestrado). Belo Horizonte: Universidade Federal de Minas Gerais, 1982.

8) Ritz V, Kortmann H, Koch K. Reduction, Softening and Melting Properties of Pellets, Sinters, Lumpy Ore and Mixed Blast Furnace Burden. In: ICSTI/Ironmaking Conference Proceedings, 1998: 1635. 
9) Iljana M, Kemppainen A, Paananen T, Mattila O, Heikkinen E, Fabritus T. Evaluating the Reduction-Softening Behaviour of Blast Furnace Burden with an Advanced Test. ISIJ Internacional. 2016, 10: 1706-1714.

10) Clixby G. Softening and melting of superfluxed sinters and acid pellets. In: $39^{\text {th }}$ Ironmaking Conference. Washington; 1980: 370-389.

11) Nishimura T, Higuchi M, Kumitomo K. ISIJ Internacional. 2011, 51: 1316.

12) Bakker $T$, Heerema $R H$. Determination of the fundamental mechanisms underlying softening and melting of blast furnace burden materials. In: ICSTI/Ironmaking Conference Proceedings, 1998: 1597-1608.

13) Nogueira PF. Blast Furnace Softening and Melting Phenomena (PhD Thesis) Carnegie Mellon Institute of Technology, The United States of America, 2003.

14) Rocha GT, Torres B, Seshadri V. Amolecimento dos constituintes da carga metálica do alto-forno. Metalurgia - ABM. 1 